\title{
Multi-Target Tracking Using a Vision Chip and its Applications to Real-Time Visual Measurement
}

\author{
Yoshihiro Watanabe, Takashi Komuro, Shingo Kagami, and Masatoshi Ishikawa \\ Department of Information Physics and Computing, University of Tokyo \\ 7-3-1 Hongo, Bunkyo-ku, Tokyo 113-8656, Japan \\ E-mail: Yoshihiro_Watanabe@ipc.i.u-tokyo.ac.jp \\ [Received October 17, 2004; accepted January 6, 2005]
}

\begin{abstract}
Real-time image processing at high frame rates could play an important role in various visual measurement. Such image processing can be realized by using a high-speed vision system imaging at high frame rates and having appropriate algorithms processed at high speed. We introduce a vision chip for high-speed vision and propose a multi-target tracking algorithm for the vision chip utilizing the unique features. We describe two visual measurement applications, target counting and rotation measurement. Both measurements enable excellent measurement precision and high flexibility because of high-frame-rate visual observation achievable. Experimental results show the advantages of vision chips compared with conventional visual systems.
\end{abstract}

Keywords: target tracking, vision chip, real-time image processing, visual measurement

\section{Introduction}

Measurement using vision is useful in wide range of applications because visual systems enable contactless measurement and highly flexible operation. In conventional vision-based systems, however, which mainly consist of cameras for imaging and computers for processing, the frame rate is limited by the video signal, for example, $33 \mathrm{~ms}$ for NTSC, which is a serious constraint on the performance of visual measurement.

Recently, a system called high-speed vision has been developed to overcome this problem. High-speed vision can overcome the frame rate limitation in conventional visual systems and enables high-speed image processing with over 1000 frames/s. Using this device, some of the advantages of real-time visual measurements become evident. For example, targets moving at high speed and with irregular motion can be measured with high precision. We can also create mechanical systems for robot control and inspection that respond to rapid changes in the motion of such objects.

In this paper, we use a so-called vision chip, which is one of the most commonly used components for implementing high-speed vision. The vision chip achieves high-speed visual feedback at $1 \mathrm{kHz}$ by integrating sensors and processors together in one chip. Therefore, as chip output, we can obtain only desired characteristics of an input image instead of the input image itself, which allows high-frame-rate imaging. Also, the vision chip has several benefits such as compactness, low cost, and low power consumption because it is a VLSI-based system. We have developed a general-purpose vision chip that consists of programmable processing elements [1].

We propose a multi-target tracking algorithm for the vision chip in this paper. This enables simultaneous observation of multiple targets at high frame rates. We develop the algorithm by adding a new labeling algorithm using binary search to a conventional tracking algorithm called self-windowing [2]. Our algorithm utilizes the unique features of the vision chip, such as high-frame-rate imaging and pixel parallel operation.

Two real-time visual measurements using the vision chip are also described. Both of them are based on multitarget tracking processing in the vision chip.

One of the applications is target counting. This application counts the cumulative number of targets coming into sight. This can also count only desired targets. At a frame rate of $0.5 \mathrm{kHz}$, we found experimentally that the maximum target speed was $20 \mathrm{~km} / \mathrm{h}$. This counting technique could be applied, for example, to high-speed inspection of small creatures and crops.

Another possible application is the measurement of high-speed rotation. In this application, the rotation axis and rotation speed of a ball are measured in real-time. As a result of experiments at $1 \mathrm{kHz}$ frame rate, the maximum rotation speed of the object was $1200 \mathrm{rpm}$. We believe that such rotation measurement could find applications in ball games, such as soccer and baseball.

In section 2 of this paper, we briefly describe the vision chip and target tracking studies. Also included in section 3 is a description of the multi-target tracking algorithm developed for the vision chip. In sections 4 and 5 , we explain the experimental system and some results of our target counting and rotation measurement algorithms, respectively. Section 6 concludes our paper. 


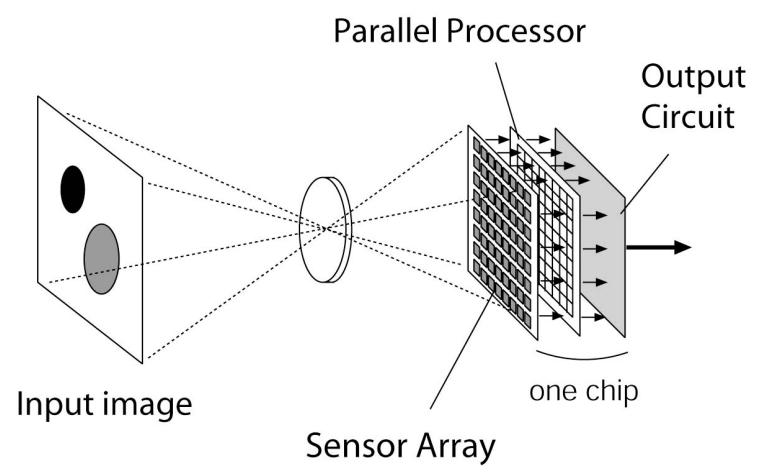

Fig. 1. Model of a vision chip [1].

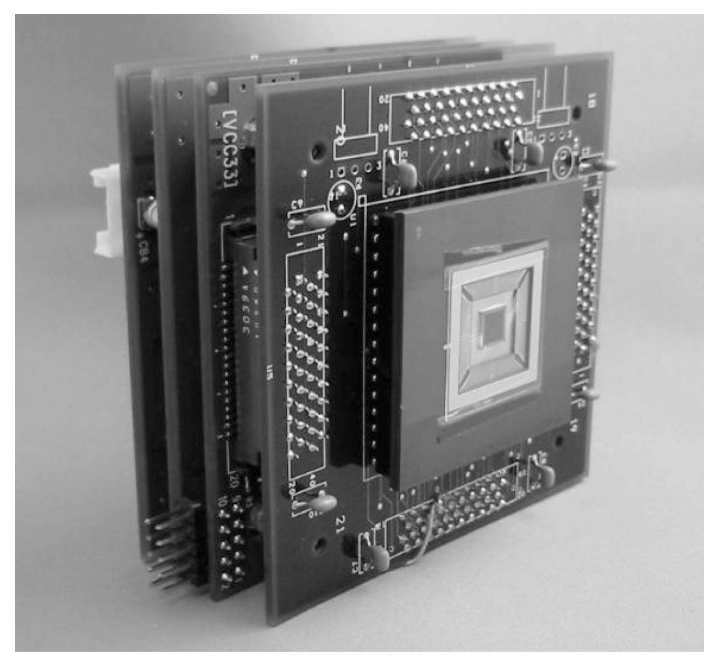

Fig. 2. General-purpose vision chip system [4].

\section{Background}

\subsection{Vision Chip}

Fig.1 shows a model of the vision chip. A vision chip is a device in which a photo detector and a processing element are provided in each pixel. Therefore, a vision chip integrates an imaging system and a processing system on one chip, systems which have been separated conventionally. This architecture provides some advantages in comparison to the traditional approach. These advantages include high-speed image processing, compactness, low power dissipation, and so on.

In this paper, a general-purpose digital vision chip system we have developed [3] is used. Fig.2 shows a photograph of this vision chip system, which consists of a chip and its controller. Resolution is $64 \times 64$. Programmable processing elements enable various image processing operations controlled by software. Also, global features such as summation and the centroid of an entire image can be extracted at high-speed. These advantages of this vision chip is thought to play an important role in practical applications.

\subsection{Target Tracking Studies}

This section describes studies about target tracking. Visual devices are classified into conventional, high-speed and VLSI-based.

In conventional visual systems, most tracking algorithms are based on prediction because of its low frame rate. Such algorithms are difficult to be used in precise tracking to targets with high-speed or irregular motion.

Among such studies, one uses a system consisting highspeed CMOS sensor and dedicated processors such as FPGA [5, 6]. However, the system is large, expensive and consumes power because it transfers and processes large amounts of data. Another system achieves image processing at high frame rates by reading out and processing only the surrounding image of a noted object [7,8], which, although promising in some applications, is unsuitable for those, for example, detecting new objects coming into sight.

In VLSI-based examples, Brajovic et al. developed a tracking computational sensor [9] with high frame rates that tracks only a single target. They also developed a sensor which allows to track multiple targets [10]. However, it cannot perform correct calculation of each target's centroid since regional division based on brightness works poorly. Komuro et al. developed a digital vision chip specialized for target-tracking [11] with the possibility of being applied to multi-target tracking, but feasible cases are few because the chip only processes matching of a target between frames and has no appropriate circuits generating new tracked targets.

\section{Multi-Target Tracking Algorithm for a Vi- sion Chip}

There has been a tracking algorithm for the vision chip called self-windowing but with tracking of only a single target, since this is based on simple matching of a tracked target. In order to track multiple targets, labeling for generating new tracked targets is needed. Also the labeling algorithm should be suitable for execution on the vision chip. Under these requirements, we propose a multi-target tracking algorithm for the vision chip, which adds a new labeling algorithm to the conventional tracking algorithm.

Our multi-target tracking algorithm utilizes the unique features of the vision chip, such as high-frame-rate imaging and pixel parallel operation. As will be shown below, these features allow the algorithm to be simplified, thus realizing high-speed processing. The input images for this algorithm are assumed to be binary.

The algorithm consists of labeling and tracking. Labeling is the initial process to generate new targets, and tracking is the process to carry out regional division and matching of each target between frames. Labeling is thus applied only to frames in which the number of targets changes and tracking is carried out for every frame, as long as the target remains in the image. The details of each process are described in the next two sections. 


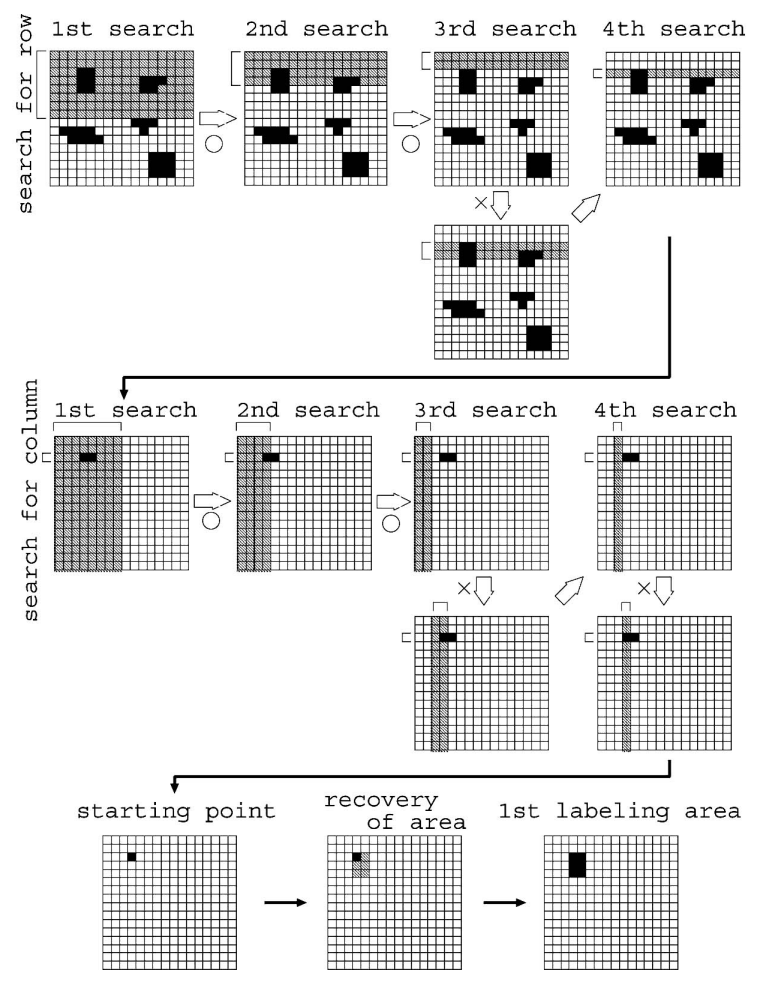

Fig. 3. Labling algorithm using binary search.

\subsection{Labeling Using Binary Search}

Conventional labeling algorithms use raster scanning, requiring large amounts of operation and not suited for a vision chip which is a parallel processing system. Nakano et al. proposed a labeling algorithm utilizing parallel operation [12], but room exists for improvement because the region search is carried out one row/column at a time.

As the labeling algorithm for our vision chip, we propose a method using binary search of an entire image. Binary search enables efficient labeling. Moreover, this labeling method is executed at high-speed by utilizing pixel parallel operation and high-speed calculation of summation, which are the unique functions of the vision chip. Fig.3 shows the flow of this labeling algorithm. In this algorithm, labeling is performed by restoring a labeled region from a point obtained by binary search. The details are below.

\section{Obtaining starting point $g_{k}^{i}=S\left(I_{k}\right)$}

- Search for a row including regions by dividing image $I_{k}$ at time $k$ into two.

- Obtain point $g_{k}^{i}$ in region $i$ by searching for the column.

- Let point $g_{k}^{i}$ obtained be the starting point of a region.

\section{Restoration of labeled region}

$$
\left\{\begin{aligned}
{ }^{0} C_{k}^{i} & =g_{k}^{i} \\
{ }^{j+1} C_{k}^{i} & =D_{1}\left({ }^{j} C_{k}^{i}\right) \cap I_{k} \quad\left(j=1,2, \ldots, n_{l}\right)
\end{aligned}\right.
$$

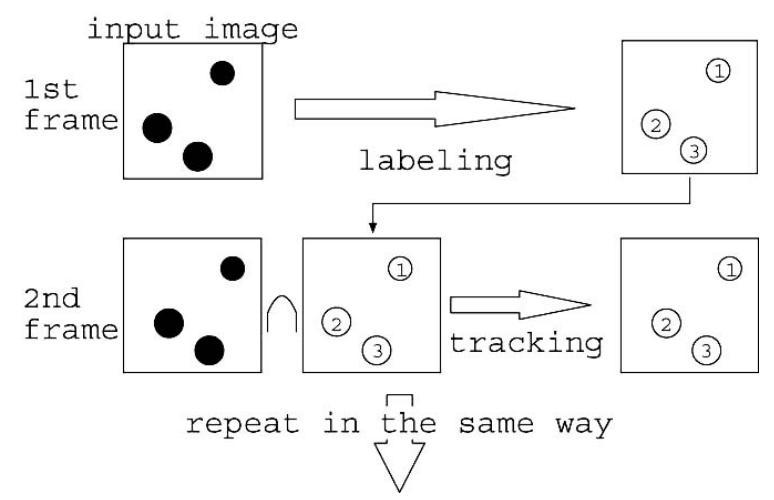

Fig. 4. Tracking.

- Restore image $C_{k}^{i}$ of region $i$ from original image $I_{k}$ and starting point $g_{k}^{i}$ by repeating 1-pixel dilation $D_{1}$ and logical multiplication $n_{l}$ times.

\section{Input image update $I_{k}^{\prime}=I_{k} \cap \overline{C_{k}^{i}}$}

- Update the image by deleting labeled region $C_{k}^{i}$ to form new input image $I_{k}^{\prime}$.

\section{Repeat}

- Repeat steps 1 to $3 M$ times

( $M$ is the number of regions to be labeled.)

In order to detect the existence of regions in search area, we use logical addition of the image, which is obtained by multiplying logically between the area and input image. This is obtained using summation calculation. Repetition number $n_{l}$ for restoration process is determined by the targets size. For example, when a target is a circle with a radius of 5 pixels, the operation must be repeated 10 times. Repetition number $M$ of a whole process is determined based on each application. Also, it is possible to label necessary targets preferentially by adding preprocessing, which leaves particular targets in an image based on features such as size and shape.

Binary search reduces the amount of operations obtaining a start point. We compare the whole operation for new labeling method to conventional method using raster scanning. When the resolution and the size of targets are set to $N \times N$ and $L$ respectively, the order of conventional operation is $O\left(N^{2} f(L)\right) . f(L)$ is the number of scanning depending on target size. In our proposed method, the number of search for obtaining a start point and the number of repetition for restoration is proportional to $\log _{2} N$ and $L$, respectively. Therefore the order is $O\left(M\left(\log _{2} N+L\right)\right)$. This means that the labeling method using binary search excels at processing speed when the resolution becomes high and the number and the size of targets are small.

\subsection{Tracking}

New tracked target regions are generated by labeling. Then, after initializing the frame, tracking is applied. We 
use a previously proposed method called self-windowing to match the target regions between frames in tracking. This novel method is based on the high-frame-rate imaging, which allows us to assume that the movement of any moving region between two successive frames is within one pixel. Under this assumption, the matching algorithm is described simply by logical multiplication and a dilation operation. Also, the pixel parallel operation enables high-speed execution. In this paper, we use a softer assumption that any moving region overlaps itself between two successive frames in order to track targets moving at high-speed. We also can track targets by repeating selfwindowing. Fig.4 shows an outline of the tracking, and actual algorithm is described in detail below.

\section{Restoration of tracked region}

$$
\left\{\begin{aligned}
{ }^{0} C_{k}^{i} & =C_{k-1}^{i} \\
{ }^{j+1} C_{k}^{i} & =D_{1}\left({ }^{j} C_{k}^{i}\right) \cap I_{k} \quad\left(j=1,2, \ldots, n_{t}\right)
\end{aligned}\right.
$$

- Restore image $C_{k}^{i}$ of tracked region $i$ at time $k$ from input image $I_{k}$ and image $C_{k-1}^{i}$ by repeating 1-pixel dilation $D_{1}$ and logical multiplication $n_{t}$ times.

\section{Repeat}

- Carry out step 1 for multiple tracking images in turn.

Number $n_{t}$ is determined by the amount of movement between frames based on the application. We believe that collision and separation between some regions, which are common problems in tracking, can be detected from changes in trajectory and area based on high-frame-rate observation, at least in simple cases.

\subsection{Algorithm Execution Time}

This section describes the execution time of the multitarget tracking algorithm on our vision chip system.

Execution time $T_{l}^{1}$ for binary search in a $N \times N$ image is $2 a \log _{2} N+b h$, when time for a single search is $a$, time for modification of search area is $b$, and the number of modifications is $h\left(0 \leq h \leq 2 \log _{2} N\right)$. Search area modification means the process applied when no targets exist in the area. Time $T_{l}^{2}$ for restoration of a region is $c n_{l}$, when time for a single restoration is $c$. Therefore, the execution time for labeling $M$ regions is $M\left(T_{l}^{1}+T_{l}^{2}\right)$. On our vision chip, parameters are as follows: $a=3.0 \mu \mathrm{s}, b=1.0 \mu \mathrm{s}$, $c=2.5 \mu \mathrm{s}, N=64$. For example, to label a circle target with a radius of 5 pixels, it takes $61-73 \mu \mathrm{s}$.

On the other hand, the execution time for tracking is $M c n_{t}$. When $n_{t}$ is 5 , this can be applied to 80 targets within $1 \mathrm{~ms}$.

There is room of improvement in current vision chips to speed up multi-target tracking. For example, we must use full adders to calculate logical addition of a whole image in labeling. This is speeded up by implementing appropriate circuits.

As shown above, real-time observation of multiple targets in image sequences at high frame rates is possible with our proposed multi-target tracking using the vision

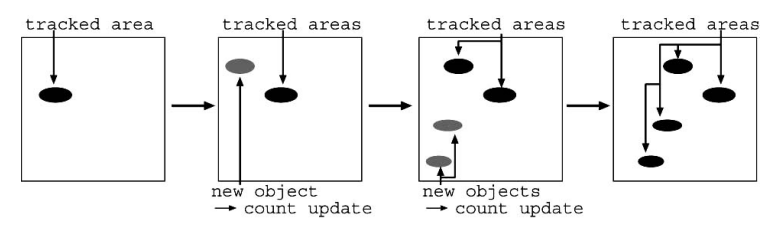

Fig. 5. Method of target counting.

chip. Visual measurements described in this paper are based on this multi-target tracking. Two applications, target counting and rotation measurement, are described in the next two sections. Both realize contactless and highspeed measurement. Also, flexible measurement can be carried out by adding suitable image processing to our programmable vision chip.

\section{Target Counting}

\subsection{Concept}

Target counting is a measurement that counts the number of regions that newly appear in an image. This counting is realized at high-frame rate by using multi-target tracking via the vision chip.

Fig.5 explains how the cumulative number is obtained by target counting. In multi-target tracking, regions in an input image are divided into two groups, new regions and tracked regions. New regions are obtained by deleting tracked regions from the input image. Counting the cumulative number is achieved by updating the result for each new region. Labeling for generating new targets in multi-target tracking is used for updating the result.

Tracking in target counting ensures the accuracy of the measurement. For example, the error that occurs when the same region is counted several times can be avoided even when many targets in the image sequences move freely. In addition, image processing on a vision chip enables target counting with desired shape and size.

This target counting enables precise measurement of objects at high-speed. For example, as an application of this target counting we consider counting small fishes swimming over a line. This kind of application is very difficult to automate because the targets move very quickly and irregularly.

\subsection{Experimental Results}

Fig.6 shows the target counting system used for this experiment. The system consists of a vision chip, its controller, and a computer. The computer obtains the counting result held in the vision chip and displays it. As targets, 100 regions are set on a rotating drum with a radius of $15 \mathrm{~cm}$. The size of the targets is about $1.0 \mathrm{~cm}$ (10 pixels) in the direction of movement. LED lighting (LDL$100 \times 100, \mathrm{CCS})$ is set inside the rotating drum and enters the vision chip directly through the pattern on the drum. 


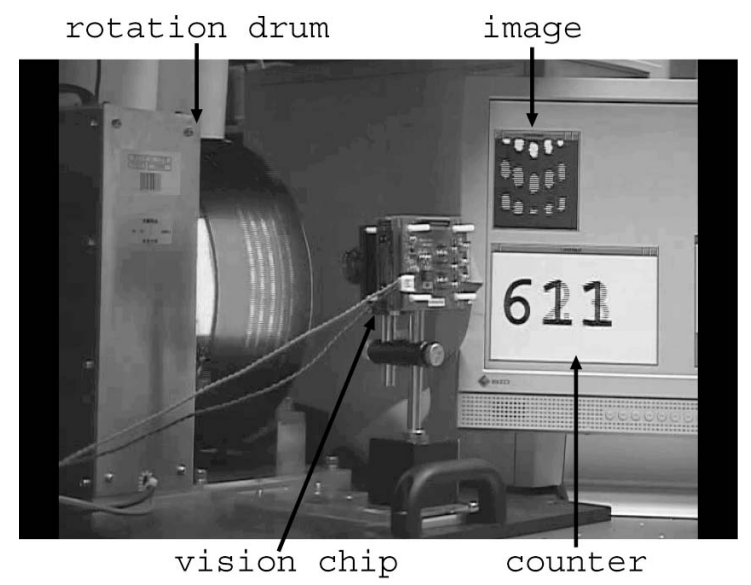

Fig. 6. Target counting system for experiment.

The cumulative number increases in cycles because target movements are controlled by the drum rotating at a constant speed. In addition, as a de-noising condition in this experiment, only targets larger than a fixed size are counted.

The frame rate is determined by the maximum number of new targets that appear in one frame. Labeling and calculation of summation are applied to new targets. In this experiment, the number is 15 . Execution time for labeling is about $100 \mu \mathrm{s}$ and time for summation is $12 \mu \mathrm{s}$ in this experiment. Therefore, we set the frame rate as $0.5 \mathrm{kHz}$.

Fig.7 shows the experimental results of target counting. Fig.7(a) is the result of counting the cumulative number when the drum rotates at $254 \mathrm{rpm}$. The result increasing periodically shows that the counting was carried out correctly.

The counting result at different speeds is evaluated in Fig.7(b). This figure compares the rotation speed of the drum, estimated from the result of the cumulative number, with the true rotation speed obtained by a motor encoder. The vertical axis in the graph is the estimated speed and the horizontal axis is the true speed. The straight line in the graph passes through the origin and the gradient is 1 . Therefore, the points on the line are accurate. As shown in Fig.7(b), the target counting is accurate up to about 350rpm.

In this experiment, the rotation speed limit of the drum is about 350rpm, which corresponds to a target speed limit of about $20 \mathrm{~km} / \mathrm{h}(5500$ pixels $/ \mathrm{s})$. This shows that our target counting can be applied to quickly moving targets. The limit occurs because the assumption in tracking that the same region overlaps with itself between frames cannot be fulfilled. Considering the application to targets moving freely, collision and separation between some regions must occur. In order to detect this problem, for example, we can use changes of each target area based on high-frame rate observation, as mentioned before.

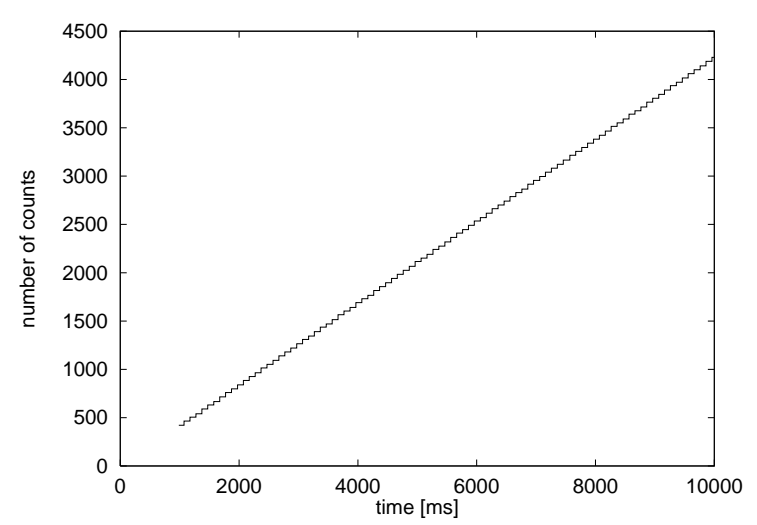

(a) Result of counting cumulative number

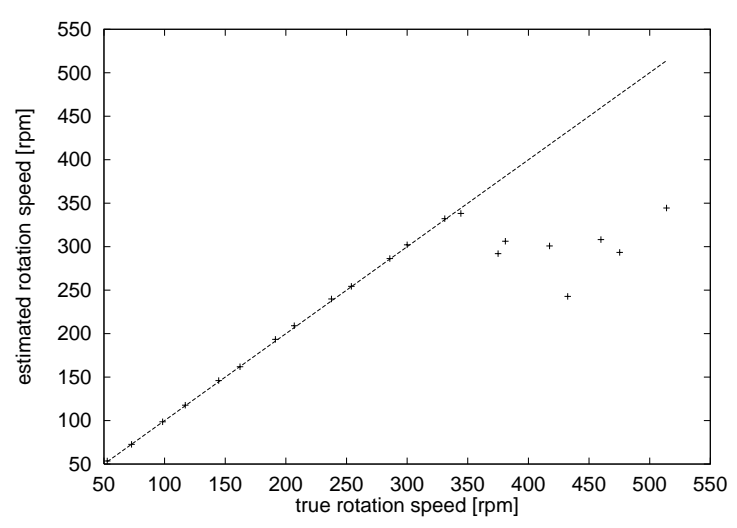

(b) Evaluation

Fig. 7. Experimental result of target counting.

\section{Rotation Measurement}

\subsection{Concept}

This rotation measurement obtains rotation information on a rotating object in real-time. The rotation information is the rotation axis and rotation speed. High-frame-rate observation enables precise measurement even when an object changes its motion quickly and randomly. In this paper, the shape of the object is spherical.

As an application of this rotation measurement, we consider ball games. For example, the rotation speed of a soccer ball in a free kick reaches about 600rpm and a baseball at pitching reaches about 1800rpm. It is difficult for conventional vision systems to realize the performance required for these applications. We also consider robot control applications based on similar measurement.

This measurement estimates the rotation information from the distribution of speed vectors on a sphere. Vectors are obtained by multi-target tracking. The method includes three processing steps as follows.

(1) Obtaining two-dimensional trajectories $\boldsymbol{P}^{i}=$ $A\left(I_{0}, I_{1}, \ldots, I_{n}\right) \quad(1 \leq i \leq m)$

- Obtain trajectories $\boldsymbol{P}^{i}$ of region $i$ on a sphere from input images. 
(2) Determining three-dimensional speed vectors $\boldsymbol{v}^{i}=$ $B\left(\boldsymbol{P}^{i}\right)$

- Determine three-dimensional speed vectors of region $i$ from two-dimensional trajectories.

(3) Estimating rotation information $[\boldsymbol{l}, \boldsymbol{\omega}]=$ $C\left(\boldsymbol{v}^{1}, \boldsymbol{v}^{2}, \ldots, \boldsymbol{v}^{m}\right)$

- Estimate rotation axis $\boldsymbol{l}$ and rotation speed $\omega$ from vectors.

Processing steps are described in the next section. The position of each target region is given by centroids of regions. Therefore, the positions can be obtained with subpixel precision.

The measurement system consists of three devices, a vision chip, its controller, and a computer, as is target counting. Processing (1) is carried out in the vision chip and its controller. Processing (2) and (3) are carried out in an external computer.

\subsection{Processing for Measurement}

In processing (1), trajectories of targets are obtained by multi-target tracking. The actual method is described in detail below.

(1-1) Labeling $\boldsymbol{C}_{0}=L\left(I_{0}\right)$

- Dividing 1st input image $I_{0}$ into $m$ target images $\boldsymbol{C}_{0}=\left[C_{0}^{1}, C_{0}^{2}, \ldots, C_{0}^{m}\right]$ by labeling.

(1-2) Tracking $C_{k}^{i}=T\left(I_{k}, C_{k-1}^{i}\right) \quad(k \geq 1)$

- Obtain image $C_{k}^{i}$ of target $i$ at time $k$ by selfwindowing between input image $I_{k}$ at time $k$ and image $C_{k-1}^{i}$ of target $i$ at time $k-1$.

(1-3) Moment extraction $\boldsymbol{M}_{k}^{i}=M\left(C_{k}^{i}\right) \quad(k \geq 1)$

- Extract 0th and 1st moments $\boldsymbol{M}_{k}^{i}$ from image $C_{k}^{i}$ of target $i$ at time $k$.

(1-4) Centroid calculation $\boldsymbol{P}_{k}^{i}=E\left(\boldsymbol{M}_{k}^{i}\right) \quad(k \geq 1)$

- Calculate centroid $\boldsymbol{P}_{k}^{i}$ of target $i$ at time $k$ from moments $\boldsymbol{M}_{k}^{i}$.

$m$ tracked targets are generated by labeling process every $n+1$ frames. Tracking and centroid calculation to these targets is applied in other frames.

In processing (2), three-dimensional speed vectors are determined from two-dimensional trajectories $\boldsymbol{P}^{i}=$ $\left[\boldsymbol{P}_{1}^{i}, \boldsymbol{P}_{2}^{i}, \ldots, \boldsymbol{P}_{n}^{i}\right]$. The details are shown below.

(2-1) Determining three-dimensional position vectors $\boldsymbol{r}_{k}^{i}=F\left(\boldsymbol{P}_{k}^{i}\right)$.

- Determine three-dimensional position vectors from the centroid of target $i$ at time $k$ using orthogonal projections and assumed spherical shape.

(2-2) Determining the three-dimensional speed vectors $\boldsymbol{v}^{i}=\frac{\sum_{k=1}^{n-1}\left(\boldsymbol{r}_{k+1}^{i}-\boldsymbol{r}_{k}^{i}\right)}{n}$

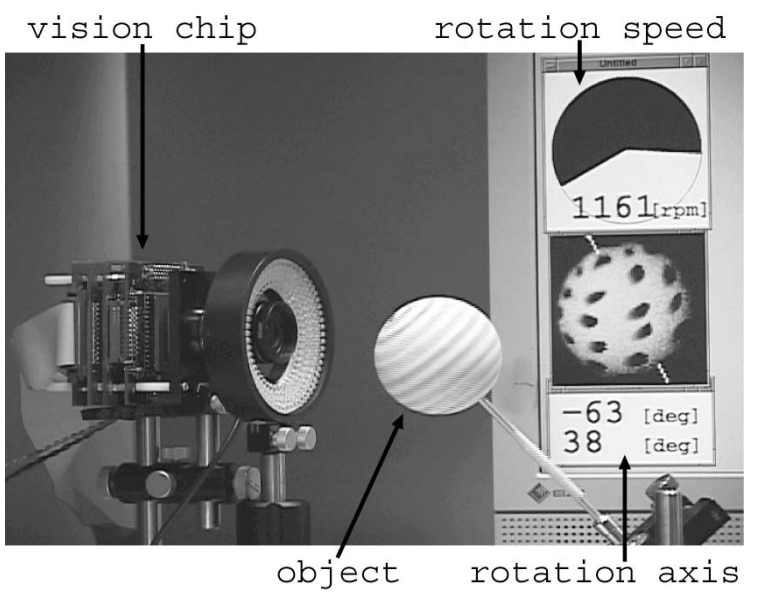

Fig. 8. Rotation measurement system for experiment.

- Determine the three-dimensional speed vectors of target $i$ at time $k$ from the motion average between frames.

In processing (3), rotation information is estimated based on the following equations. The center of the sphere is assumed to be the origin. The origin corresponds to the center of an image. The imaging area is parallel to the $x y$ plane. A sphere is imaged from positive direction $z$.

Rotation axis $l$ is obtained from eq.(1) using the least squares method. Speed vector $\boldsymbol{v}$, position vector $\boldsymbol{r}$ of each target, and radius $R$ of the sphere in an image are needed for this equation. Radius and correction for the sphere center are obtained from the input image.

$$
l_{x} v_{x}+l_{y} v_{y}-\frac{r_{x} v_{x}+r_{y} v_{y}}{\sqrt{R^{2}-r_{x}^{2}-r_{y}^{2}}}=0
$$

Rotation speed $\omega$ is approximately calculated by eq.(2). This equation needs speed vector $\boldsymbol{v}_{0}$ at position $\boldsymbol{r}_{0}$ and distance $h$ between the origin and the plane passing through the position $\boldsymbol{r}_{0}$ and orthogonal to the rotation axis $\boldsymbol{l}$. The equation assumes that $\boldsymbol{v}_{0}$ is small enough.

$$
\omega=\frac{\left|v_{0}\right|}{\sqrt{R^{2}-h^{2}}}
$$

\subsection{Experimental Results}

Fig.8 shows the rotation measurement system used in the experiment. A rotating sphere is illuminated by LED lighting (IRDR-90, NISSIN). Also, it is assumed that only a single rotating sphere is imaged on the screen and the background has no other objects. A pattern is applied to the sphere for easy extraction of target regions from an image.

The number of tracked targets is 10 and the number of positions for obtaining the trajectory is 10 . These numbers are equivalent to $m$ and $n$ in section 5.1, respectively. Both $m$ and $n$ must be at least 2 in order to obtain a solution. Greater accuracy is achieved with higher values. 

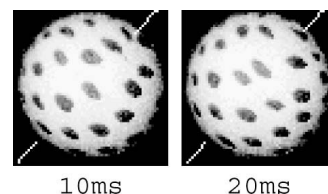

$20 \mathrm{~ms}$

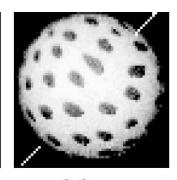

$30 \mathrm{~ms}$

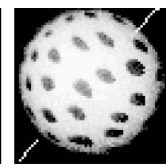

$40 \mathrm{~ms}$

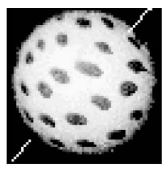

$50 \mathrm{~ms}$

Fig. 9. Image sequences of measured rotation axis.

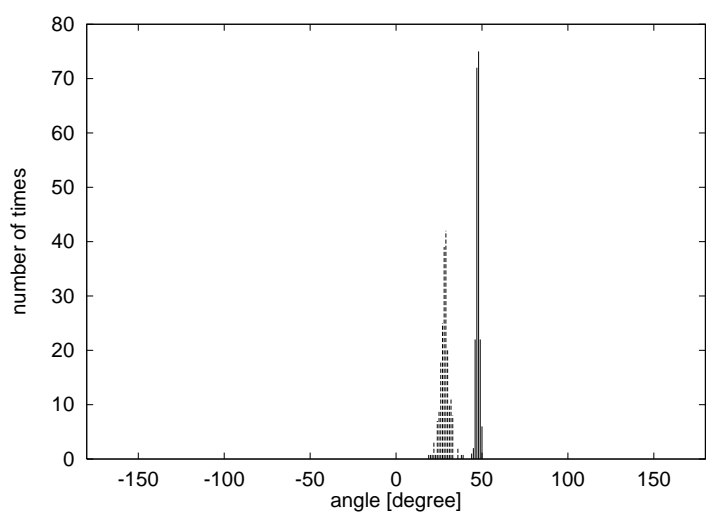

(a) Distribution of rotation axis

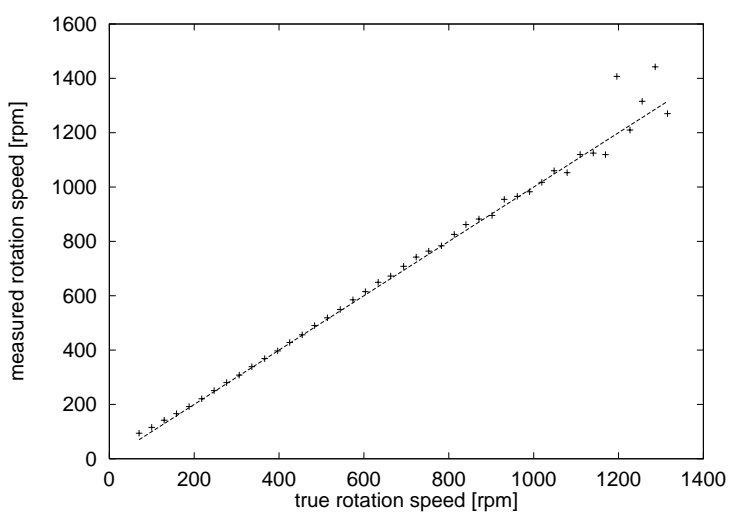

(b) Evaluation of rotation speed

Fig. 10. Experimental result of rotation measurement.

Labeling takes about $100 \mu$ s per single target in this experiment. Tracking and centroid calculation per single target takes about $12 \mu \mathrm{s}$ and $58 \mu \mathrm{s}$, respectively. Therefore we set the frame rate as $1 \mathrm{kHz}$ at all sequences equally.

The results obtained when the rotation axis is defined by $\theta \approx 45^{\circ}, \gamma \approx 30^{\circ}$ and the rotation speed is fixed at 335rpm are shown in Figs.9 and 10(a). $\theta$ is the angle of the rotation axis in the $x y$ plane. $\gamma$ is the angle made by the rotation axis and the $x y$ plane. Fig.9 shows image sequences of measured targets; the rotation axes are drawn in the images.

Fig.10(a) shows the distribution of the rotation axis measured 200 times. The histogram in solid lines is the result for $\theta$ and that in dashed lines is for $\gamma$. The experimental conditions were the same as in Fig.9. The results are distributed centered on a fixed angular value. As shown in this figure, the rotation axis was measured almost correctly, considering that the axis can only be fixed roughly and the rotation axis of the observed object is not completely stable because of the experimental environment.

Fig.10(b) shows evaluation of the measured rotation speed. This figure compares the results to the true speed. The vertical axis is measured value and the horizontal axis is the true one. Plotted points are the experimental results. The straight line in the graph passes through the origin and the gradient is 1. As shown in Fig.10(b), rotation measurement is accurate up to about 1200rpm.

The 1200-rpm limit occurs for the same reason as the limit in target counting. The result shows that this system can measure rotation information even of an object rotating at high speed.

Considering the application to the ball games, in particular, soccer, this system enables measurement of a ball during a free kick, whose rotation speed is about 600rpm. On the other hand, in baseball, when the ball is pitched, its rotation speed is about 1800rpm, which is difficult to be measured in this experimental condition. However, such measurement is possible in our system because the frame rate can be improved by reducing the number of tracked regions $m$. We also improve the frame rate by designing processing elements of the vision chip to be specialized for multi-target tracking. In addition, there are problems existing with extraction of a complex pattern on a normal ball and application to a ball flying through the air as remain to be solved.

\section{Conclusion}

We describe the algorithm of multi-target tracking for the vision chip. This allows us to realize real-time observation of multiple targets at high frame rates in the compact system. Also, as its applications, we describe two visual measurements based on multi-target tracking, target counting and rotation measurement. The experimental results using the vision chip we have developed show that both methods excel in the points, high-precision, highframe-rate observation and flexibility. These visual measurements, providing such advantages, are expected to be applied in various fields, such as inspection, industrial applications, sport measurement, robot control, and so on.

\section{References:}

[1] T. Komuro, S. Kagami, and M. Ishikawa, "A New Architecture of Programmable Digital Vision Chip," Proceeding of 2002 Symposium on VLSI Circuits, pp. 266-269, 2002.

[2] I. Ishii, and M. Ishikawa, "Self Windowing for high speed vision," Proceedings of 1999 IEEE International Conference on Robotics and Automation, pp. 1916-1921, 1999.

[3] S. Kagami, T. Komuro, I. Ishii, and M. Ishikawa, "A Real-Time Visual Processing System using a General-Purpose Vision Chip," Proceedings of 2002 IEEE International Conference on Robotics and Automation, pp. 1229-1234, 2002.

[4] http://www.k2.t.u-tokyo.ac.jp/vision/index-e.html

[5] R. Okada, J. Oaki, D. Yamamoto, N. Kondoh, and J. Amemiya, "High-speed Object Tracking in Ordinary Surroundings Based on Temporally Evaluated Optical Flow," Proceedings of 2003 IEEE/RSJ International Conference on Intelligent Robots and Systems, pp. 242-247, 2003. 
[6] Y. Nakabo, M. Ishikawa, H. Toyoda, and S. Mizuno, "1ms Column Parallel Vision System and its Application of High Speed Target Tracking," Proceedings of 2000 IEEE International Conference on Robotics and Automation, pp. 650-655, 2000.

[7] K. Tajima, A. Numata, and I. Ishii, "Development of a Highresolution, High-speed Vision System using CMOS Image Sensor Technology Enhanced by Intelligent Pixel Selection Technique," Proceedings of SPIE Vol.5603, pp. 215-224, 2004.

[8] U. Muehlmann, M. Ribo, P. Lang, and A. Pinz, "A New High Speed CMOS Camera for Real-Time Tracking Applications," Proceedings of 2004 IEEE International Conference on Robotics and Automation, pp. 5195-5200, 2004.

[9] V. Brajovic, and T. Kanade, "Computational Sensor for Visual Tracking with Attention," IEEE Journal of Solid-State Circuits, Vol.33, No.8, pp. 1199-1207, Aug. 1998.

[10] V. Brajovic, "An Object Tracking Computational Sensor," tech. report CMU-RI-TR-01-40, Robotics Institute, Carnegie Mellon University, December 2001.

[11] T. Komuro, I. Ishii, M. Ishikawa, and A. Yoshida, "A Digital Vision Chip Specialized for High-speed Target Tracking," IEEE transaction on Electron Devices, Vol.50, No.1, pp. 191-199, 2003.

[12] T. Nakano, T. Morie, M. Nagata, and A. Iwata, "A CellularAutomaton-Type Image Extraction Algorithm and Its Implementation Using an FPGA," Proceedings of IEEE Asia-Pacific Conference on Circuits and Systems (APCCAS 2002), Vol.2, pp. 197-200, 2002.

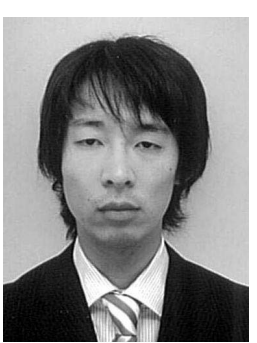

Name:

Yoshihiro Watanabe

\section{Affiliation:}

Doctor Course Student, Department of Information Physics and Computing, Graduate School of Information Science and Technology, University of Tokyo

Address:

7-3-1 Hongo, Bunkyo-ku, Tokyo 113-8656, Japan

\section{Brief Biographical History:}

2004 Received M.E. degree in Department of Information Physics and Computing from the University of Tokyo, Tokyo, Japan

2004- Doctor Course Student, Department of Information Physics and Computing, University of Tokyo

2004- Research Fellow, Japan Science and Technology Corporation

Membership in Learned Societies:

- The Robotics Society of Japan (RSJ)

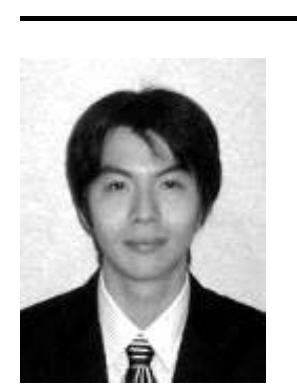

Name:

Takashi Komuro

\section{Affiliation:}

Research Associate, Department of Information Physics and Computing, Graduate School of Information Science and Technology, University of Tokyo

Address:

7-3-1 Hongo, Bunkyo-ku, Tokyo 113-8656, Japan

Brief Biographical History:

2001 Received Ph.D. degree in Mathematical Engineering and Information Physics from University of Tokyo, Tokyo, Japan

2001-2002 Research Fellow, Japan Science and Technology Corporation 2002- Research Associate, Research Associate, Department of Information Physics and Computing, University of Tokyo

Main Works:

- "Dynamically Reconfigurable SIMD Processor for a Vision Chip," IEEE Journal of Solid-State Circuits, Vol.39, No.1, pp. 265-268, 2004.

- "A Digital Vision Chip Specialized for High-speed Target Tracking," IEEE transaction on Electron Devices, Vol.50, No.1, pp. 191-199, 2003.

- "Device and System Development of General Purpose Digital Vision Chip," Journal of Robotics and Mechatronics, Vol.12, No.5, pp. 515-520, 2000.

\section{Membership in Learned Societies:}

- The Robotics Society of Japan (RSJ)

- The Society of Instrument and Control Engineers (SICE)

- The Institute of Image Information and Television Engineers (ITE) 


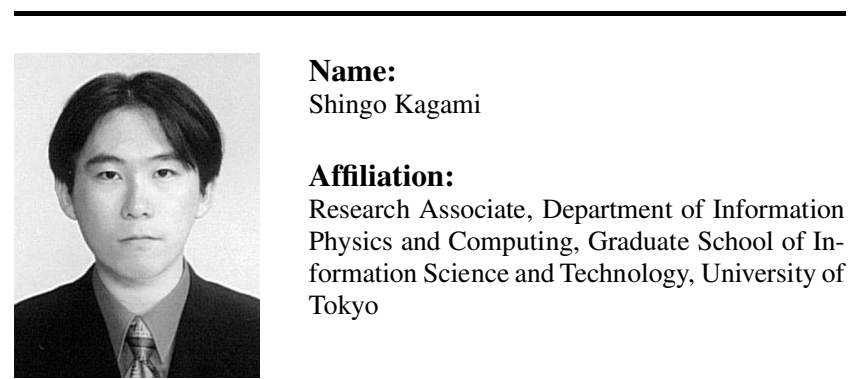

Address:

7-3-1 Hongo, Bunkyo-ku, Tokyo 113-8656, Japan

Brief Biographical History:

2003 Received Ph.D. degree in Mathematical Engineering and Information Physics from the University of Tokyo, Tokyo, Japan

2003 Research Fellow, Japan Science and Technology Corporation

2003- Research Associate, Department of Information Physics and

Computing, University of Tokyo

Main Works:

- "A Sensor Selection Method Considering Communication Delays," Proc.

IEEE Int. Conf. Robotics and Automation, pp. 206-211, Apr. 2004.

Membership in Learned Societies:

- The Robotics Society of Japan (RSJ)

- The Institute of Image Information and Television Engineers (ITE)

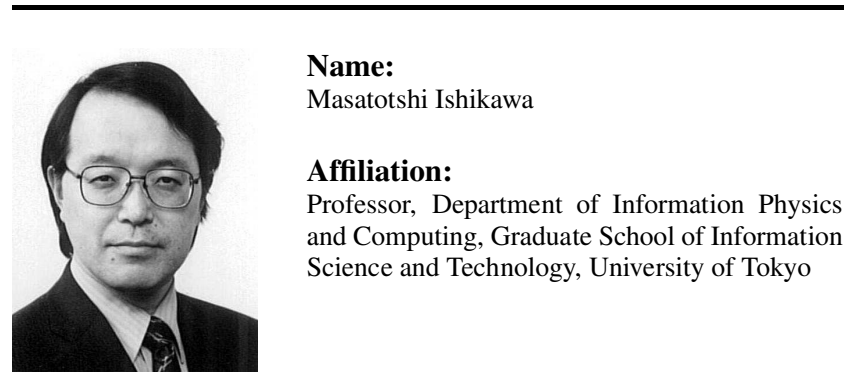

Address:

7-3-1 Hongo, Bunkyo-ku, Tokyo 113-8656, Japan

Brief Biographical History:

1979- Senior Researcher, Industrial Products Institute

1989- Associate Professor, University of Tokyo

1999- Professor, University of Tokyo

Main Works:

- "A CMOS Vision Chip with SIMD Processing Element Array for 1ms

Image Processing," Proc. IEEE Int. Solid-State Circuits Conference

(ISSCC'99), pp. 206-207, Feb. 1999.

Membership in Learned Societies:

- The Robotics Society of Japan (RSJ)

- The Society of Instrument and Control Engineers (SICE)

- The Institute of Electronics, Information and Communication Engineers

(IEICE) 